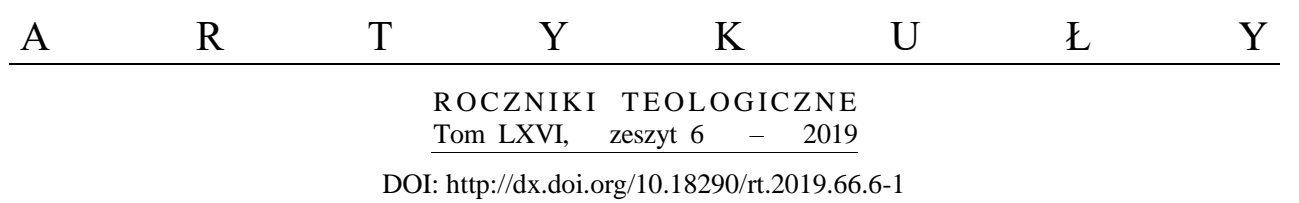

MICHAL KAPLÁNEK

\title{
INKULTURATION UND CHARISMA. ÜBERLEGUNGEN ZUR EINHEIT IN DER VERSCHIEDENHEIT
}

\author{
INCULTURATION AND CHARISMA. \\ REFLECTIONS ON UNITY IN DIVERSITY
}

\begin{abstract}
A b stract. The author's, a Czech Salesian opening concept is the original effect of the God's incarnation which had an impact on the history of Jewish nation. Christianity was injected into the specific socio-cultural stream of Hellenistic (Greek), Jewish thought. All three missionary actions were marked by being imbued with the current forms of thought and custom. In the $20^{\text {th }}$ century this form of adaptation is called inculturation. This is to say that the Son of God was born into a specific socio-economic environment and not some abstract reality. This is the foundation of Christian faith in all cultures and movements known as "Young Churches." The author, however expands this view of missionary concepts wider than that in Ad Gentes. In his article he explains the meaning of inculturation for the current and post-modern scenes. He also supports the concept for the sake of finding new "ways of life" deriving from the original idea to be applicable in other countries and continents.
\end{abstract}

Key words: inculturation; missionary activity; charism; modernity; postmodernism.

Dr hab. Michal KAPLÁNEK, prof. USB - Katedra Edukacji, Wydział Teologii, Uniwersytet Południowoczeski w Czeskich Budziejowicach; adres do korespondencji: ul. Kněžská 8, CZ-370 01 České Budějovice, Czechy; e-mail: kaplanek@tf.jcu.cz; ORCID iD: https://orcid. org/0000-0001-7200-6813

Assoc. Prof. Dr. Michal KAPláneK - Department of Education, Faculty of Theology, University of South Bohemia in České Budějovice; address for correspondence: Kněžská 8, CZ-370 01 České Budějovice, Czech Republic; e-mail: kaplanek@tf.jcu.cz; ORCID iD: https:// orcid.org/0000-0001-7200-6813 


\section{INKARNATION UND UNIVERSALITÄT}

Die Offenbarungslehre, die innerhalb der Fundamentaltheologie weiterentwickelt wurde, geht davon aus, dass der einzige Gott, der Schöpfer des Universums, sich in der Konkretheit der Geschichte dem Menschen offenbart. Während das Judentum - trotz des Universalitätsanspruchs der Autorität Gottes - eine Stammes - oder Nationalreligion geblieben ist, wurde das Christentum von Anfang an zu einer Missionsreligion, d.h. zu einer Religion, die sich als universeller Heilsweg versteht. Der Widerspruch zwischen der Universalität des Heilsangebots Gottes und der Diversität der Kulturen und Religionen wurde schon im Judentum als Geheimnis des Unheils verstanden (vgl. Gen 11, 1-9). Die Geschichte über den Turmbau zu Babel ist eine theologische Interpretation der Diversität: Der Hochmut des Menschen sei die Ursache der Missverständnisse, sogar der Unmöglichkeit des Miteinanders zwischen Kulturen und Nationen. Schon die Tatsache, dass diese Geschichte zum Bestandteil der biblischen Urgeschichte wurde, ist ein Beweis für die Reflexion dieses Widerspruchs im althebräischen Denken.

Im Judentum wurde der Heilswille Gottes, zumindest in der älteren Geschichte (vor Jesaja), auf das auserwählte Volk reduziert. Das mag die Folge der kulturellen Bedingungen sein, die das Überleben und Fortdauern der mosaischen Religion eigentlich nur in der Form einer Nationalreligion erlaubten. Doch schon die Entstehung des nachexilischen Judentums zeigt einen bedeutsamen Unterschied zu anderen Nationalreligionen jener Zeit. Die Entstehung der Thora - in der heutigen Form - ermöglichte eine gewisse Abkoppelung des Judaismus von Palästina und dadurch auch sein Überleben in einer transformierten Gestalt. Die jüdische Religion konnte dann - wenn auch in einer eingeschränkten Form - ebenso außerhalb von Judäa und Israel praktiziert werden. Weitere Geschichten des hellenistischen Judentums beweisen die Existenz von mehreren kulturellen Formen der jüdischen Religion. Die griechische Übersetzung von Thora, Newiim und Ketuwim sowie das Einschließen der original griechischen Texte in die Septuaginta, bedeuteten den Anfang der Inkulturation des Judentums in die griechische Welt. Doch auch die jüdischen Diaspora-Gemeinden wollten keine volle Integration in die „heidnische Kultur“. Diese Distanzierung des Judaismus von den religiösen und kulturellen Gewohnheiten vor Ort wurde durch die Koppelung der Religion mit der Identität Israels als auserwähltem Volk abgesichert. Proselytismus war eine Ausnahmeerscheinung. Durch die Judenverfolgung im Römischen Reich, die Zerstörung des Jerusalemer Tempels durch Titus 
und die Trennung der frühen Kirche von der Synagoge wurde die Koppelung der jüdischen Religion mit der ethnischen Identität der Juden verstärkt. Die Absonderung der Juden in der späteren „,christlichen“ Geschichte führte zu einem verstärkten Identifizierungsmodell zwischen der mosaischen Religion und der jüdisch-ethnischen Identität.

An der theologischen Entwicklung, die in den sog. deuterokanonischen Schriften gut $\mathrm{zu}$ beobachten ist, ist ein bedeutsamer Einfluss der griechischen Philosophie auf das Judentum zu erkennen: die abstrakte Betrachtungsweise der zwischenmenschlichen Beziehungen, der Gedanke der unsterblichen Seele, die nach dem biologischen Tod ein weiteres Leben führt und viele andere Themen.

\section{INKULTURATION JUDENTUMS UND CHRISTENTUMS IN EINE HELLENISTISCHE PHILOSOPHIEGESCHICHTE}

Die Entstehung der griechischen Version der hebräischen Bibel (Septuaginta) war der erste Schritt für die Inkulturation des späteren Judentums in die hellenistische Kultur, obwohl bereits die sog. deuterokanonischen Schriften - wie etwa die Makkabäerbücher - den Konflikt zwischen dem Judentum und dem heidnischen Hellenismus darstellten. Doch auch das spätere Judentum bekam durch die messianische Bewegung, die etwa in Deuterojesaja zum Vorschein kommt, universalistische Züge. Dadurch wurde die Entstehung der Gruppen von Proselyten oder "Gottesfürchtigen“ überhaupt erst ermöglicht. Auch wenn die Juden keine aktive Missionstätigkeit betrieben, gab es immer wieder Menschen, die aus irgendeinem Grund zum „erwählten Volk“ gehören wollten. Sie wurden zwar unter den Juden nie gleichberechtigt wahrgenommen und behandelt, doch partizipierten sie am Glauben an die Verheißungen Gottes.

Die Situation des frühen Christentums war jedoch ganz anders. Die Anzahl der palästinischen Juden, die Christen wurden, war im Vergleich zu anderen Volksgruppen von Beginn an ziemlich gering, und seit der Zerstörung Jerusalems (70 n. Chr.) kann eigentlich kaum noch von neuen, aus Palästina stammenden Judenchristen gesprochen werden. Der größte Missionar der frühen Kirche, der H1. Paulus, war selbst ein Diasporajude, obwohl er nach der Ausbildung bei Gamaliel in Jerusalem zur Gruppe der Pharisäer gehörte. $\mathrm{Zu}$ seinem Missionsgebiet gehörten vor allem die jüdischen Diasporagemeinden in Kleinasien und Griechenland. 
Die Entstehung der ersten theologischen Schriften in der Form von Apologien (etwa Adversus haereses von Irenäus) war ein weiterer und bedeutsamer Schritt zur Inkulturation des Evangeliums in die römisch-griechische Gedankenwelt. Die weitere Entwicklung des Christentums verlief in dieser Linie. Doch die Christianisierung Europas im frühen Mittelalter wäre ohne die Anpassung der kirchlichen Disziplin und der „christlichen Frömmigkeit“ an die bereits vorherrschenden Umstände undenkbar gewesen. Eine interessante Zusammenfassung der Inkulturation des Christentums in drei bedeutsamen Wellen der Missionsgeschichte gab der päpstliche Prediger Raniero Cantalamessa in seinem Buch Come la scia del vascelo (Wie die Spur eines Schiffes $)^{1}$. Er beschreibt, dass die Mission von Anfang an mit Inkulturation rechnete, auch wenn dieser Begriff erst im Rahmen der Missionswissenschaft des 20. Jahrhunderts definiert wurde ${ }^{2}$.

\section{MISSIONEN UND INKULTURATION}

Innerhalb der Kirchengeschichte werden drei wichtige Missionswellen unterschieden. Die erste ist die Verbreitung des Christentums im antiken Römischen Reich und parallel in den anderen Kulturen, angefangen von Syrien und Äthiopien über Persien bis hin zu Indien. Auf dem Gebiet des heutigen Europa hat sich seit Ende des 1. Jahrhunderts die hellenisierte Form des Christentums verbreitet und weiterentwickelt. Obwohl Jesus und seine direkten Jünger palästinische Juden (aus Judäa oder Galiläa) waren, wurde durch das Netz, das Paulus bei seiner Mission aufbaute, Kontakt zu Diasporajuden hergestellt ${ }^{3}$. Die Bibel der Christen - zumindest seit Ende des 1. Jahrhunderts - war nicht der hebräische Tanach (Thora, Newiim, Ketuwim), sondern die griechische Septuaginta. Daneben sind die neutestament-

\footnotetext{
${ }^{1}$ Cantalamessa Raniero, Come la scì di un vascello. Orizzonti per una nuova evangelizzazione (Cinisello Balsamo: Ed. San Paolo, 2012).

2 Zum ersten Mal wurde der Begriff „Inkulturation“ vom Jesuiten Joseph P. Masson 1959 verwendet („L'Église ouverte sur le monde“. Nouvelle revue théologique 84(1962):1032-1043). In den päpstlichen Dokumenten ist dieser Begriff zum ersten Mal im Apostolischen Schreiben Catechesi tradendae von Johannes Paul II zu finden (Art. 53).

3 Die Unterschiede zwischen dem hellenistischen und hebräischen Judentum werden im christlichen Kontext im Zusammenhang mit der Geschichte des Christentums des 1. Jahrhunderts und vor allem mit dem Wirken des Apostels Paulus untersucht. Dazu z. B. Paulus und das antike Judentum, Hrsg. Martin Hengel und Ulrich Heckel (Tübingen: Mohr, 1991) oder in der tschechischen theologischen Literatur: Mireia Ryšková, Pavel z Tarsu a jeho svět [Paulus von Tarsus und seine Welt] (Praha: Karolinum, 2014).
} 
lichen Schriften nur im griechischen Text erhalten geblieben, obwohl einige von ihnen (etwa das Matthäusevangelium) eine direkte Bindung zur hebräischen Tradition darstellen. Auf jeden Fall ist die erste Missionswelle durch die Anpassung des Christentums an die griechisch-römische Denkweise gekennzeichnet.

Der sog. Sacco di Roma (Plünderung der Stadt Rom durch die westgotischen Krieger im Jahre 410) wurde von vielen für das „Ende der Welt“ gehalten. Nur der H1. Augustinus sah die Zukunft in einem „Gottesstaat“ („civitas Dei“), der jene „civitas terrena“ ersetzen sollte. Daher waren die Geschehnisse seiner Zeit für ihn „kein Ende der Welt, sondern nur das Ende einer Welt"“4. In der Zeit nach dem Zerfall des Weströmischen Reichs (476) änderte sich auch langsam das Verhältnis der Christen zu den „Barbaren“. Sie wurden für „potenzielle Brüder im Glauben“ gehalten ${ }^{5}$. Seit der Konfrontation der antiken Kirche mit den Völkern des damaligen Europa kann von der Christianisierung (West-Europas) gesprochen werden. Die Christianisierung Europas stellt die zweite Missionswelle der Kirchengeschichte dar. Ohne Zweifel passte sich das Christentum an die Denkweise und Bräuche dieser Nationen teilweise an.

Ganz anders verlief die dritte Missionswelle, die mit der Kolonialisierung Amerikas und Afrikas verbunden war. Alle Teile Amerikas wurden missioniert und gleichzeitig einer der europäischen Mächte als Kolonien zugeteilt (Spanien, Portugal, England). Cantalamessa beschreibt die Umstände Anfang des 16. Jahrhunderts sehr realistisch und stellt schließlich fest: „Nur ein paar große Geister, allen voran die Dominikaner Antonio von Montesino und Bartolomeo de Las Casas, hatten den Mut, sich gegen die Missbräuche der Eroberer (conquistatori) und für die Verteidigung der Rechte der Eingeborenen auszusprechen“6. Diese Verschmelzung von Missionierung und Kolonialismus kann zwar historisch erklärt, jedoch nicht verteidigt werden. Als Reaktion hierauf entstand in der zweiten Hälfte des 20. Jahrhunderts eine tiefgreifende Skepsis gegenüber der Missionstätigkeit der Kirche(n), insbesondere in Bezug auf die hochentwickelten Kulturen Asiens. Die Antwort des Magisteriums auf die berechtigte Kritik an neuzeitlichen Missionsstrategien ist im Dekret Ad gentes des 2. Vatikanischen Konzils zu finden. Die Mission stellt - nach dem Dekret Ad gentes - nicht mehr nur eine Teilaufgabe der Kirche dar, sondern ist zu ihrer Kernaufgabe geworden: Kirche ist „ihrem

\footnotetext{
${ }^{4}$ Cantalamessa, Come la scìa di un vascello, 22-23.

${ }^{5}$ Ebd. 23.

${ }^{6}$ Ebd. 38.
} 
Wesen nach missionarisch“7 . Missionen sind weder bloße Mittel der „Propaganda fidei“, noch nur Strukturen für die humanitäre Entwicklungshilfe.

Wie die Mission selbst theologisch begründet und verankert ist, so muss auch die Inkulturation als Leitlinie für heutige kulturgerechte Verkündigung theologisch verankert werden. Die Inkulturation ist nicht nur eine pastorale Strategie, sondern Ausdruck des Inkarnationsprinzips. Derselbe Gottessohn, der in die konkreten soziokulturellen Umstände hineingeboren ist, stellt auch das Fundament des Glaubens der Christen aller Völker und Nationen dar, einschließlich der sog. ,jungen Kirchen“:

In der Tat nehmen die jungen Kirchen, verwurzelt in Christus, gebaut auf das Fundament der Apostel, nach Art der Heilsordnung der Fleischwerdung in diesen wunderbaren Tausch alle Schätze der Völker hinein, die Christus zum Erbe gegeben $\operatorname{sind}^{8}$.

Die Inkulturation setzt sogar die Entstehung (neuer) Theologien voraus,

die Gottes Offenbarung, wie sie in der Schrift bezeugt und in der Tradition ausgelegt wird, mit der Philosophie und Weisheit der Völker, mit ihren Lebensformen und ihrem Ethos ,vermitteln', indem sie die Konvergenzen und die Zugänge zum Evangelium, die sich von dorther ergeben, aufarbeiten. [...] Damit wird die Einheit der Kirche notwendigerweise zu einer Einheit in Vielheit ${ }^{9}$.

Es ist verständlich, dass der Weg der Inkulturation auch manche Risiken in sich birgt. Daher wurde im 22. Artikel des Dekrets vor Synkretismus bzw. Partikularismus gewarnt. Doch die Kirche geht - seit dem 2. Vatikanum diesen Weg, der sich als berechtigt ausweist.

Obwohl die Aufarbeitung des Begriffs Inkulturation vor allem im Rahmen der Missionstheologie vorangetrieben wurde, ist dieses Prinzip im gesamten kirchlichen Leben als praktische Folge der Inkarnation gegenwärtig.

\footnotetext{
${ }^{7}$ AG 2.

${ }^{8}$ AG 22.

${ }^{9}$ Peter Hünermann, ,Theologischer Kommentar zum Dekret über die Missionstätigkeit der Kirche Ad gentes“, 298, in Herders theologischer Kommentar zum Zweiten Vatikanischen Konzil, Bd. 4 (Freiburg, 2005): 219-336.
} 


\section{DAS 2. VATIKANISCHE KONZIL: INKULTURATION IN DIE MODERNE}

Einen ebenso großen Schritt wie bei der Anpassung der Theologie auf die Kulturen der indigenen Völker in Afrika und Lateinamerika, machte die Kirche im Vorfeld des 2. Vatikanischen Konzils im Blick auf die Kulturwende in Europa und Nordamerika, die seit der Aufklärung begonnen hatte und nach Ende des 2. Weltkriegs unwiderruflich vollendet wurde. Es handelte sich um eine tiefgreifende Veränderung der Denkweise und des Verhältnisses zu religiösen Traditionen bei einer gleichzeitig ständig wachsenden europäischen Bevölkerung, vor allem in hoch industriellen Ländern. Es wird auch über einen Einstieg in die Moderne gesprochen. Mit dem Begriff „,modern" wird alles verbunden, was mit dem wissenschaftlichen und technischen Fortschritt der Neuzeit zusammenhängt, sowie die politischen und philosophischen Ideen, die den demokratischen Staaten der Gegenwart zugrunde liegen und sich größtenteils an die Tradition der Aufklärung anschließen. Mit der schnellen Entwicklung von Wissenschaft und Technik in den letzten zwei Jahrhunderten wurde der Glaube mit den unbegrenzten Möglichkeiten menschlicher Vernunft verbunden: Man glaubte, der Mensch sei fähig, sein Leben und die Kräfte der Natur so zu beherrschen, dass es in der Zukunft kein Problem mehr geben würde, das für den Menschen unlösbar wäre.

Die katholische Kirche wehrte sich seit der Französischen Revolution gegen alle politischen, kulturellen und philosophischen Strömungen, die den traditionellen Status quo der europäischen Gesellschaften relativieren oder sogar radikal ändern wollten. Daher beobachtete die Theologie die Demokratisierungstendenzen in der Gesellschaft sowie den entstehenden Rationalismus bei den Philosophen und Theologen mit einer tiefgreifenden Skepsis. Zugleich kam es aber in vielen europäischen Ländern zu einer radikalen soziokulturellen Wende von der traditionellen Gesellschaft mit klar konturierten und in manchen Bereichen von der Kirche bestimmten Lebensregeln zu einer Gesellschaft, die durch eine Ideologie (des Nationalismus bzw. Sozialismus) beseelt war. Durch ihre Verteidigungsstrategie (Apologetik) verlor die katholische Kirche ihre bisherige Stellung in der Gesellschaft und - in wichtigen Segmenten der Bevölkerung - auch ihre Alltagsrelevanz. Als Reaktion auf den Säkularisierungsprozess wurden jene Tendenzen verstärkt, die aus der Kirche eine „Festung“ (in sich eingeschlossene „communio“) machen wollten. Es entstand eine Kluft zwischen der Kirche und den (laizistischen) Gesellschaften und Kulturen, die immer tiefer wurde. 
Die Gefahr der Isolierung der Kirche von den breiten Bevölkerungsschichten und eine wachsende Entfremdung der neuscholastischen Theologie von der Entwicklung der Philosophie weckten in einigen Priestern, Theologen und katholischen Denkern Reformanstöße, die aber bis in die 50er Jahre des 20. Jahrhunderts als „modernistisch“ disqualifiziert wurden. Trotzdem entstanden Reformbewegungen (Bibelbewegung, Liturgiebewegung, Laienapostolat), die mit Recht als „Vorreiter des 2. Vatikanums“ bezeichnet werden ${ }^{10}$.

$\mathrm{Zu}$ den wichtigsten Ergebnissen des Zweiten Vatikanischen Konzils gehört die Versöhnung zwischen der katholischen Kirche und der Moderne, die am stärksten in der Pastoralkonstitution Gaudium et spes zum Vorschein kommt. Obwohl alle lehramtlichen Dokumente der Kirchengeschichte kontextuell gelesen werden müssen, ist die enge und explizite Verbindung des Texts mit der konkreten historischen Situation für Gaudium et spes so kennzeichnend, dass sie zu einem Verbindlichkeitsproblem seitens der Konzilskritiker führte. In Gaudium et spes wurde die Verteidigungsstrategie der katholischen Kirche gegenüber der Moderne durch eine Offenheit zur „Welt von heute" ersetzt. Die Vermächtnisse der philosophischen Entwicklung nach der Aufklärung - wie die Religionsfreiheit und Demokratie - werden seit dem 2. Vatikanum nicht mehr als gefährlich angesehen, sondern als eine berechtigte Art von menschlicher Autonomie anerkannt.

Doch bereits in den Jahrzehnten nach dem Konzil brachen die Zukunftshoffnungen der Moderne, die auf dem Glauben an die menschliche Vernunft und den immerwährenden Fortschritt der Wissenschaft und Politik aufgebaut worden waren, zusammen. Manche sprechen über eine neue Epoche, die sog. Postmoderne. Andere lehnen diesen Begriff ab, weil sie in den charakteristischen Zügen unserer Zeit keine neue Werte entdecken können, sondern nur die Negation oder Überwindung der bisherigen Werte. Als charakteristisch für die letzten Jahrzehnte des 20. Jahrhunderts galten die folgende Merkmale: die Pluralität der Weltanschauungen und Lebensstile, die Relativierung der Vernunft, die hypothetisch-reflexive Einstellung zur Realität, die Entmächtigung des Subjekts, die Macht der Bilder und die Auflösung des Identitätskonzeptes ${ }^{11}$. Gerade die Unbestimmtheit der Lebenskonzepte in der Zeit der Pluralität erwirkt bei vielen unserer Zeitgenossen eine diffuse Unsicher-

\footnotetext{
${ }^{10}$ Zur Situation der Kirche vor dem 2. Vatikanischen Konzil gibt es zahlreiche Publikationen - im Tschechischen z. B. Tomáš Petráček, Církev, tradice, reforma (Praha, Vyšehrad, 2016).

${ }^{11}$ Vgl. Werner Helsper, "Jugend im Diskurs von Moderne und Postmoderne", 17-22, in: Jugend zwischen Moderne und Postmoderne, Hrsg. Werner Helsper (Opladen, 1991), 11-38.
} 
heit, derer manche Menschen durch den Rücktritt in die bewahrte traditionalistische Praxis entkommen wollen ${ }^{12}$.

\section{INKULTURATION IN DIE POSTMODERNE?}

Die bisher beschriebenen Modelle der Inkulturation des Christentums innerhalb der Weltgeschichte mögen den Eindruck erwecken, dass es bei der Inkulturation um eine Anpassung und Applikation des Christentums in ein geschlossenes System ginge. Doch die Realität war (und ist) anscheinend anders. Weder das Diasporajudentum als Missionsfeld des Hl. Paulus noch die bereits entstehenden europäischen Staten bildeten geschlossene Systeme. Umso weniger darf gegenwärtig die sog. Postmoderne als ein geschlossenes und genau definierbares System bezeichnet werden. Auch die Lehre der Kirche kann nicht in einer solchen Definitionen eingeschlossen werden, obwohl die Notwendigkeit, die Glaubensartikel - in der Konfrontation mit Irrlehren - zu definieren, immer wieder entsteht. Alle lehramtlichen Definitionen sind immer nur Versuche, das Undefinierbare (das „ganz Andere") in die Worte zu fassen, um dadurch den Glauben dialog- und streitfähig zu machen. Alle Dogmenformeln stellen nämlich Inkarnationen von Glaubenswahrheiten in die jeweilige philosophische Denkweise dar. Darum wäre es illusorisch, nach dem Vorbild der Neuscholastik, aus den Worten (der Bibel oder der Dogmen) eine „reine Essenz“ herauskristallisieren zu wollen.

Die Inkulturation ist nämlich keine Umsetzung „,reiner Wahrheiten“ in einen relativen Kontext, sondern eher eine Übersetzung des gesamten Gedankenguts, das in einem kulturellen Kontext verankert wurde, in einen anderen kulturellen Kontext. Daher muss die Arbeit eines Theologen, der das christliche Gedankengut in einen neuen Kontext ,übersetzen“ will, der Arbeit eines Dolmetschers ähneln. So wie ein Dolmetscher die Denkweise der Sprecher beider zu übersetzenden Sprachen verstehen muss, so muss auch der Theologe den „Sitz im Leben“ der Kirche in der Entstehungszeit einer lehramtlichen Aussage gut kennen und zugleich die Spezifika der konkreten Kultur, in die es zu übersetzen gilt, verstehen.

\footnotetext{
${ }^{12}$ Zum Verhältnis zwischen der scheinbaren Sicherheit des kommunistischen Regimes und der gegenwärtigen Unsicherheit in den postkommunistischen Ländern vgl. Michal Kaplánek, „Von bescheidener Sicherheit zur unsicherer Freiheit“, Concilium 54(2018): 198-206.
} 


\section{DIE CHARISMEN DER ORDEN UND BEWEGUNGEN VOR UND NACH DER WENDE IN MITTEL- UND OSTEUROPA (1989)}

Innerhalb des Christentums und vor allem innerhalb der katholischen Kirche entwickelten sich in der Geschichte zahlreiche Formen der konkreten Lebensweise, die meistens aus dem Charisma des Gründers/der Gründerin heranwuchsen. Es wird dann von Charismen oder „Spiritualitäten“ einzelner Institute oder Bewegungen gesprochen. Doch jedes Institut und jede Bewegung entstand in einer konkreten Zeit, in einem konkreten Land; ihre Gründer waren Personen mit natürlichen Begabungen und (oft ganz besonderen) Charismen. Darum halte ich es für angebracht auch über die „Inkulturation von Charismen“" zu sprechen. Das Konzilsdekret Perfectae caritatis beinhaltet zwar keines von diesen zwei Wörtern, doch der Sinn des Dekrets besteht in der „Anpassung des Ordenslebens an die Erfordernisse unserer Zeit“13.

Nach dem Konzil wurden die Ordensregeln aller Institute neu gefasst und neue Ausbildungsordnungen herausgearbeitet. Vielen Mühen wurden dem Studium der „eigentlichen Absichten der Gründer“ ${ }^{14}$ gewidmet. Doch bereits ein Gründer oder eine Gründerin war das Kind seiner/ihrer Zeit und seines/ihres Landes. Insbesondere die apostolischen Orden wurden sehr stark durch praktische Lebensweisen des Ursprungslandes beeinflusst. Die Spiritualität eines Ordens oder einer Gemeinschaft entsteht nicht durch die Applikationen theoretischer Grundsätze, sondern durch die praktische Einführung konkreter Lebensweisen. Sowie in jeder Familie wird die Geborgenheit eines Zuhauses in den alltäglichen Kleinigkeiten spürbar wird, wächst auch eine Gemeinschaft durch gemeinsame Erlebnisse und Rituale erst richtig zusammen.

Wenn etwa ein Oratorium der Salesianer Don Boscos ${ }^{15}$ besucht wird, das in der ersten Hälfte des 20. Jahrhunderts aufgebaut wurde, ist stets auf dem Hof des Hauses ein großer Spielplatz, von dem das Haus durch Arkaden erreichbar ist, vorzufinden. Im Laubengang ist immer ein Wasserhahn installiert, um den Durst der stetig laufenden Jungen zu stillen. Dieser Baustil ist nicht nur in Italien, dem Ursprungsland der Salesianer, sondern in allen Salesianerhäusern der Welt verbreitet. Die Arkaden sind für Norditalien sehr

\footnotetext{
${ }^{13}$ Perfectae caritatis, 18 .

${ }^{14}$ Perfectae caritatis, $2 \mathrm{~b}$.

${ }^{15}$ Der Verfasser ist Mitglied der Ordensgemeinschaft der Salesianer Don Boscos (SDB). Er ist in diese Gemeinschaft in der Zeit des Kommunismus (1988) eingetreten. Dies war damals nur inoffiziell (im Geheimen) möglich.
} 
praktisch, sogar - wegen dem häufigen Regen - fast unverzichtbar. Doch in anderen Ländern, wo mehr Schutz vor der Kälte als vor dem Regen benötigt wird, werden die Arkaden weniger genutzt und haben auch wenig Sinn. Es gibt also zahlreiche Bräuche oder kleine Rituale, die einen geschichtlichen Ursprung in einem konkreten Land und in einer konkreten Zeit hatten, aber in anderen Kontexten ihren Sinn verlieren.

Das Charisma eines Instituts ist sicher in seiner Tradition, angefangen vom Gründer/von der Gründerin, festgelegt, aber nicht ausgeschöpft. Das Charisma drückt sich in verschiedenen Kontexten von Neuem aus. Es entstehen ganz neue Ausdrucksformen, die manchmal in andere Kontexte nicht transferiert werden können. Daher gehört es zu den Aufgaben der Leitung der Ordensinstitute „die Lebensverhältnisse der Menschen, die Zeitlage sowie die Erfordernisse der Kirche“ zu beobachten ${ }^{16}$, um das Charisma in verschiedenen Kontexten konkret zu erfüllen.

Die Geschichte der Kirche in der Zeit des kommunistischen Regimes verbirgt (noch) nicht genug entdeckte und erforschte Chancen und Herausforderungen in sich. In den Kirchen des ehemaligen Ostblocks wird die eigene Vergangenheit nur als Herausforderung, nicht als Chance wahrgenommen. In der Zeit der kommunistischen Unterdrückung funktionierte in manchen Ländern (z. B. in der ehem. Tschechoslowakei) eine regelmäßige Kirchenstruktur nur begrenzt. Es gab keine kirchlichen Schulen oder Caritaswerke; Ordensgemeinschaften durften keine neuen Mitglieder aufnehmen, es gab auch keine Männerklöster mehr. Wenn die Christen auf die wesentlichen Formen des Kirchenlebens (Orden, Bewegungen) nicht ganz verzichten wollten, mussten sie das kirchliche Leben an die politische Situation anpassen. Dadurch entstanden neue Formen des kirchlichen Lebens, die zwar dem Text des Kodex des kanonischen Rechts nicht genau entsprachen, jedoch den Geist des Kirchenrechts erfüllten. Konkret ging es um die inoffizielle Ausbildung von Priestern, Ordensleuten und ständigen Diakonen oder um geheime Gottesdienste, die ohne die Genehmigung des Staates gefeiert wurden. Bei all diesen Aktivitäten beschränkten sich die Christen auf den wesentlichen Inhalt der Lehre oder der Liturgie, ohne Äußerlichkeiten und Formalitäten. Dadurch sensibilisierten sich die Christen bereits für das Wesentliche. Zwischen Priestern und Laien entstanden informelle Beziehungen, die eine gute Basis für eine Zusammenarbeit bildeten. Die Ordensleute konnten kein normales Gemeinschaftsleben führen; kamen aber oft und gerne zusammen.

\footnotetext{
${ }^{16}$ Perfectae caritatis, $2 \mathrm{~d}$.
} 
Diese Art und Weise des Ordenslebens, die an die Situation der Verfolgung angepasst worden war, forderte die Ordensleute zu einer stärkeren eigenen Verantwortung auf, als es in den regulär lebenden Gemeinschaften in der „freien Welt" der Fall war. Dies bezog sich auf alle wesentliche Merkmale des Ordenslebens, vor allem auf die Armut, denn der Mitbruder/die Mitschwester war für die Bescheidenheit des eigenen Lebens und die Solidarität mit den Armen selbst verantwortlich. Auch in anderen Bereichen des Ordenslebens war keine Kontrolle von außen möglich. Nur die Selbstkontrolle und aufrichtige Berichterstattung an den Oberen dienten als Garantie für das Einhalten der Ordensregeln.

Nach der Wende (1989) kam es zu einer „Normalisierung“ des kirchlichen Lebens in den postkommunistischen Ländern. Dieser Prozess war zwar notwendig, verlief aber ohne eine kritische Auseinandersetzung mit dem soziokulturellen Wandel, der inzwischen in den mittel- und osteuropäischen Gesellschaften zustande gekommen war. Aus Sicht der Kirchenleitung sowie der Leitung der einzelnen Institute wurden die Notmaßnahmen, die in der Zeit des Kommunismus praktiziert worden waren, nur negativ bewertet. Die neuen Impulse, die damals entstanden waren, wurden „,vergessen“. Die Erfahrungen der geheimen Kirchenstrukturen (wie die tschechische Ecclesia silentii) wurden missachtet und die mutigen Experimente im Stillen verurteilt ${ }^{17}$. Auf ähnliche Art und Weise wurden auch in der Zeit des Kommunismus entwickelte ,alternative Formen“ der Liturgie oder der Ordenspraxis nur als mangelhaft oder sogar als abwegig beurteilt. In der Tat wäre es bereichernd, diese Formen genauer zu untersuchen, damit die Kirche von heute die wertvollen Früchte des Zeugnisses der „damaligen Christen“ (1948-1989) nicht für immer verliert. Auch dies ist eine Art der Inkulturation des Christentums.

\section{EINE ABSCHLIEßENDE ÜBERLEGUNG}

Allzu oft begegnet man heute Bischöfen, Priestern oder Theologen, die mit den befreienden Ansichten von Papst Franziskus unzufrieden sind. Der Grund ihrer Unzufriedenheit liegt wahrscheinlich darin, dass sie vom Papst erwarten, er solle die ewigen „Wahrheiten“ die im Prinzip „klar“ seien, nur

\footnotetext{
${ }^{17} \mathrm{Zu}$ diesen Experimenten gehörten nämlich die geheimen Priesterweihen von verheirateten Männern oder auch der Frauen. Vgl. Petr Fiala und Jiří Hanuš, Skrytá cirkev [Die verborgene Kirche] (Brno, 1999).
} 
applizieren, ohne jede Rücksicht auf Situation oder Person. Wenn aber der Papst sagt, die lebendigen Menschen seien mehr als tote Prinzipien, ist ein Konflikt bereits vorprogrammiert. Der Hintergrund des Konflikts besteht darin, dass die mit dem Papst unzufriedenen Theologen, die Formulierungen, die in einem gewissen historischen Kontext entstanden, für absolut gültig halten. Die Treue zum Evangelium bedeutet jedoch nicht an den einmal verlautbarten Formulierungen festzuhalten, sondern die Bedeutung des Evangeliums für heute zu entdecken. Doch dies ist nur in konkreten Kontexten möglich. Es geht nämlich darum, das ganze Evangelium in die Denkweise einer konkreten Zeit und eines konkreten Landes zu „übersetzen“, und nicht nur vor Jahrhunderten formulierte Prinzipien zu applizieren.

Es ist mir wohl bewusst, dass diese Fragen strittig sind. Doch wenn wir zurück zu den Wurzeln gehen, finden wir - auch in der Kirche von heute eine gemeinsame Basis, die es uns ermöglicht, wieder sachliche Gespräche zu führen und dadurch Polaritäten zu überwinden.

\section{LITERATUR}

Bardy, Gustave. Menschen werden Christen. Das Drama der Bekehrung in den ersten Jahrhunderten. Freiburg, Basel, Wien, 1988.

Cantalamessa, Raniero. Come la scìa di un vascello. Orizzonti per una nuova evangelizzazione. Cinisello Balsamo, 2012.

Fiala, Petr und Hanuš Jiří. Skrytá církev [Die verborgene Kirche]. Brno, 1999.

Helsper, Werner. „Jugend im Diskurs von Moderne und Postmoderne“. In Jugend zwischen Moderne und Postmoderne, Hrsg. Werner Helsper, 11-38. Opladen, 1991.

Hünermann, Peter. „Theologischer Kommentar zum Dekret über die Missionstätigkeit der Kirche »Ad gentes«". In Herders theologischer Kommentar zum Zweiten Vatikanischen Konzil, Bd. 4, 219-336. Freiburg, 2005.

Kaplánek, Michal. „Von bescheidener Sicherheit zur unsicherer Freiheit“. Concilium 54(2018): 198-206.

Masson, Joseph. „L’Église ouverte sur le monde”. Nouvelle Revue Théologique 84(1962): 10321043.

Paulus und das antike Judentum. Hrsg. Hengel, Martin und Heckel, Ulrich. Tübingen, 1991.

Petráček, Tomáš. Církev, tradice, reforma. Praha, Wyšehrad, 2016.

Ryšková, Mireia. Pavel z Tarsu a jeho svět [Paulus von Tarsus und seine Welt]. Praha, 2014. 


\title{
INKULTURACJA I CHARYZMA. ROZWAŻANIA O JEDNOŚCI W RÓŻNORODNOŚCI
}

\author{
Streszczenie
}

Punktem wyjścia rozważań autora, czeskiego salezjanina, jest wcielone działanie Boga, które wywarło wpływ na historię narodu żydowskiego. Chrześcijaństwo zostało „wszczepione” społeczno-kulturowo w hellenizowany judaizm. Wszystkie trzy fale misji w historii chrześcijaństwa naznaczone były także różnorodnym dostosowaniem doktryny i praktyki wiary do obecnych form myślenia. W teologii XX wieku do tej adaptacji użyto terminu „inkulturacja”. Inkulturacja jest rozumiana jako wyraz zasady wcielenia w działaniu Boga. Ten sam Syn Boży, urodzony w konkretnych okolicznościach społeczno-kulturowych, stanowi też fundament wiary chrześcijan wszystkich ludów i narodów, w tym także tak zwanych młodych Kościołów. Autor rozszerza jednak ważność wcielenia daleko poza granice misji ad gentes. W tym artykule wyjaśnia znaczenie inkulturacji dla nowoczesności i postmodernizmu, a także opowiada się za uwzględnianiem zasady inkulturacji w rozpowszechnianiu już sprawdzonych i poszukiwaniu nowych duchowych dróg (sposobów życia) ze społeczno-kulturowego środowiska założyciela (zakonu lub wspólnoty życia) do innych krajów i na inne kontynenty.

Słowa kluczowe: inkulturacja; działalność misyjna; charyzmat; nowoczesność; postmodernizm.

\section{INKULTURATION UND CHARISMA. ÜBERLEGUNGEN ZUR EINHEIT IN DER VERSCHIEDENHEIT}

\section{Zus a m menfassung}

Der Ausgangspunkt der Überlegungen des Verfassers, eines tschechischen Salesianers, ist die inkarnatorische Handlungsweise Gottes, die sich in der Geschichte des jüdischen Volks auswirkte. Das Christentum wurde soziokulturell auf das hellenisierte Judentum ,eingepflanzt“. Auch alle drei Missionswellen in der Geschichte des Christentums wurde durch die mannigfaltige Anpassung der Glaubenslehre und -praxis auf die aktuellen Denkformen gekennzeichnet. In der Theologie des 20. Jahrhunderts wurde dann für diese Anpassung der Begriff „Inkulturation“ gewendet. Die Inkulturation wird als ein Ausdruck des Inkarnationsprinzips der Handlung Gottes verstanden. Derselbe Gottessohn, der in die konkreten soziokulturellen Umstände hineingeboren ist, der stellt auch den Fundament Glaubens der Christen aller Völker und Nationen dar, einschließlich der sog. ,jungen Kirchen“. Der Verfasser erweitert aber die Geltung der Inkarnation über die Grenze der Missionen ad gentes weit hinaus. Er erklärt in diesem Artikel die Bedeutung der Inkulturation an die Moderne und Postmoderne und plädiert auch für die Wahrnehmung des Inkulturationsprinzips auch bei der Verbreitung der schon bewährten sowie der neuen spirituellen Wege (der Lebensweisen) aus dem soziokulturellen Milieu des Gründers/der Gründerin in andere Länder und Kontinente.

Schlüsselwörter: Inkulturation; Missionstätigkeit; Charisma; Moderne; Postmoderne. 